\title{
Investigational Product Return Record
}

National Cancer Institute

\section{Source}

National Cancer Institute. Investigational Product Return Record. NCI Thesaurus. Code C115566.

Documentation providing information regarding investigational product (IP) inventory returns to or from a distribution center, depot, and/or trial site. 